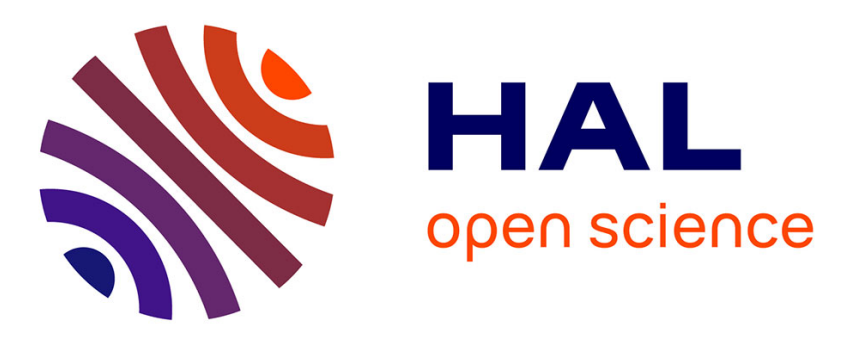

\title{
A Note on Learning Dependence Under Severe Uncertainty
}

Matthias M.C.M Troffaes, Frank Coolen, Sébastien Destercke

\section{To cite this version:}

Matthias M.C.M Troffaes, Frank Coolen, Sébastien Destercke. A Note on Learning Dependence Under Severe Uncertainty. International Conference on Information Processing and Management of Uncertainty in Knowledge-Based Systems (IPMU 2014), Jul 2014, Montpellier, France. pp.498-507. hal-01045006

\section{HAL Id: hal-01045006 https://hal.science/hal-01045006}

Submitted on 2 Sep 2014

HAL is a multi-disciplinary open access archive for the deposit and dissemination of scientific research documents, whether they are published or not. The documents may come from teaching and research institutions in France or abroad, or from public or private research centers.
L'archive ouverte pluridisciplinaire HAL, est destinée au dépôt et à la diffusion de documents scientifiques de niveau recherche, publiés ou non, émanant des établissements d'enseignement et de recherche français ou étrangers, des laboratoires publics ou privés. 


\title{
A Note on Learning Dependence Under Severe Uncertainty
}

\author{
Matthias C. M. Troffaes ${ }^{1}$, Frank P. A. Coolen ${ }^{1}$, and Sébastien Destercke ${ }^{2}$ \\ 1 Durham University, UK \\ 2 Université de Technologie de Compiègne, France
}

\begin{abstract}
We propose two models, one continuous and one categorical, to learn about dependence between two random variables, given only limited joint observations, but assuming that the marginals are precisely known. The continuous model focuses on the Gaussian case, while the categorical model is generic. We illustrate the resulting statistical inferences on a simple example concerning the body mass index. Both methods can be extended easily to three or more random variables.
\end{abstract}

Keywords: bivariate data, categorical data, copula, Gaussian copula, robust Bayesian, imprecise probability

\section{Introduction}

Sklar's theorem [10] states that any multivariate distribution of $k$ variables can be expressed through a density on $[0,1]^{k}$ with uniform marginals - this density is called a copula - and the marginal distributions of each of the variables. For this reason, copulas [7] have become an indispensible tool to model and learn statistical dependence in multivariate models: they allow of estimation of the dependence structure, separately from the marginal structure.

Estimating dependence requires joint observations, which in many cases are only available in small amounts, while substantial amounts of marginal data may be available. For example, when studying the reliability of a system, it is common to have good information about the reliability of each system component, yet to have only little information about joint failures [11]. Imprecise probabilities provide one possible theoretical basis for dealing with small sample sizes, by representing knowledge as a set of distributions [3,13,1], rather than a single distribution necessarily based on somewhat arbitrary assumptions [6].

Copulas and imprecise probabilities have been studied in the literature by various researchers. The Fréchet-Hoeffding copula bounds, which represent completely unknown dependence, are used for instance in probabilistic arithmetic [16] and p-boxes $[4,12]$. One theoretical difficulty is that there is no straightforward imprecise equivalent of Sklar's theorem, say, expressing any set of joint distributions as a sets of copulas along with a set of marginal distributions [8]: it appears that, when working with sets of distributions, separating the dependence structure from the marginal structure is a lot more difficult in general. 
In this paper, we propose and investigate a few statistical models for robust dependence learning from limited data. We state an imprecise version of Sklar's theorem when marginal distributions are fully known, separating precise marginals from the imprecise dependence structure. We propose a range of parametric models for the bivariate categorical case. Finally, demonstrate our findings on a toy example: estimating the body mass index from height and mass data.

Section 2 explores a continuous model, focusing on the multivariate normal model, while section 3 provides a first exploration of a generic categorical model.

\section{Robust Bayesian Correlation Learning for Bivariate Normal Sampling}

We start with revising a simple and well-studied model: sampling from the bivariate normal distribution. We will derive some new results that are relevant to dependence learning. Our analysis starts from Quaeghebeur and De Cooman's robust Bayesian framework for sampling from the exponential family [9].

\subsection{Inference with known Mean and Unknown Covariance Matrix}

Let $Z_{i}:=\left(Z_{i 1}, \ldots, Z_{i k}\right)$ be a multivariate normally distributed random variable with known mean - which we can assume to be zero without loss of generality through translation of the data-but unknown covariance matrix $\Sigma \in \mathbb{R}^{k \times k}$. A particular realisation of $Z_{i}$ is denoted by a lower case letter $z_{i}:=\left(z_{i 1}, \ldots, z_{i k}\right) \in$ $\mathbb{R}^{k}$. The likelihood of an i.i.d. sample $z_{1}, \ldots, z_{n}$ is:

$$
\begin{aligned}
f\left(z_{1}, \ldots, z_{n} \mid \Sigma\right) & \propto|\Sigma|^{-n / 2} \prod_{i=1}^{n} \exp \left(-\frac{1}{2} z_{i}^{T} \Sigma^{-1} z_{i}\right) \\
& =|\Sigma|^{-n / 2} \exp \left[-\frac{1}{2} \sum_{i=1}^{n} \operatorname{tr}\left(z_{i} z_{i}^{T} \Sigma^{-1}\right)\right]
\end{aligned}
$$

where the data $z_{i} \in \mathbb{R}^{k}$ are organised as row vectors, so $z_{i} z_{i}^{T}$ is the matrix containing $z_{i \ell} z_{i \ell^{\prime}}$ in row $\ell$ and column $\ell^{\prime}$.

A family of conjugate priors for this density is the family of inverse Wishart distributions with hyperparameters $\nu_{0}>0$ and $\Psi_{0} \in \mathbb{R}^{k \times k}$ positive definite [2]:

$$
f\left(\Sigma \mid \nu_{0}, \Psi_{0}\right) \propto|\Sigma|^{-\frac{\nu_{0}+k+1}{2}} \exp \left[-\frac{1}{2} \operatorname{tr}\left(\Psi_{0} \Sigma^{-1}\right)\right]
$$

The posterior distribution is obtained by updating the hyperparameters through:

$$
\nu_{n}=\nu_{0}+n \quad \Psi_{n}=\Psi_{0}+\sum_{i=1}^{n} z_{i} z_{i}^{T}
$$


The prior expected covariance matrix is given by

$$
\mathrm{E}\left(\Sigma \mid \nu_{0}, \Psi_{0}\right)=\frac{\Psi_{0}}{\nu_{0}-k-1}=: \Sigma_{0}
$$

and therefore, through conjugacy, the posterior expected covariance matrix is

$$
\begin{aligned}
\mathrm{E}\left(\Sigma \mid z_{1}, \ldots, z_{n}, \nu_{0}, \Psi_{0}\right) & =\mathrm{E}\left(\Sigma \mid \nu_{n}, \Psi_{n}\right)=\frac{\Psi_{n}}{\nu_{n}-k-1} \\
& =\frac{\Psi_{0}+\sum_{i=1}^{n} z_{i} z_{i}^{T}}{\nu_{0}+n-k-1} \\
& =\frac{\left(\nu_{0}-k-1\right) \Sigma_{0}+\sum_{i=1}^{n} z_{i} z_{i}^{T}}{\nu_{0}+n-k-1}=: \Sigma_{n}
\end{aligned}
$$

For robust Bayesian analysis aiming to learn about the dependence between two random variables, we now need to identify a reasonable set of prior distributions, or, in our conjugate setting, a reasonable set of hyperparameters $\nu_{0}$ and $\Psi_{0}$.

The formula for the posterior expected covariance matrix shows that $\nu_{0}$ determines our learning speed, that is, how many observations $n$ we need before starting to move towards our data. So, $\nu_{0}$ is similar to the $s$ value in the imprecise Dirichlet model [14]. Here too, we will simply assume $\nu_{0}$ to be fixed to whatever value is judged to lead to a reasonable learning speed. For fixed $\nu_{0}$, any particular choice of $\Psi_{0}$ corresponds to a prior covariance matrix $\Sigma_{0}$.

Let us now study the bivariate case $(k=2)$ in more detail. We will write $X_{i}$ for $Z_{i 1}$ and $Y_{i}$ for $Z_{i 2}$. We would choose

$$
\Psi_{0}=\left(\nu_{0}-3\right)\left[\begin{array}{cc}
\sigma_{X}^{2} & \rho_{0} \sigma_{X} \sigma_{Y} \\
\rho_{0} \sigma_{X} \sigma_{Y} & \sigma_{Y}^{2}
\end{array}\right]
$$

if we had prior variances $\sigma_{X}>0$ and $\sigma_{Y}>0$ for the two components, as well as the prior correlation coefficient $\rho_{0} \in[-1,1]$. For this paper focusing on dependence, we are mostly interested in cases where the marginals are well known, i.e. well known prior $\sigma_{X}$ and $\sigma_{Y}$, but unknown prior correlation $\rho_{0}$. We will therefore study the set of priors with all parameters fixed, except for $\rho_{0}$, which we assume to be vacuous a priori. Without loss of generality, by rescaling, we can assume that $\sigma_{X}=\sigma_{Y}=1$, leaving us with just two hyperparameters: $\nu_{0}>0$ and $\rho_{0} \in[-1,1]$.

The posterior covariance matrix becomes:

$$
\Sigma_{n}=\frac{1}{\nu_{0}^{\prime}+n}\left[\begin{array}{cc}
\nu_{0}^{\prime}+\sum_{i=1}^{n} x_{i}^{2} & \nu_{0}^{\prime} \rho_{0}+\sum_{i=1}^{n} x_{i} y_{i} \\
\nu_{0}^{\prime} \rho_{0}+\sum_{i=1}^{n} x_{i} y_{i} & \nu_{0}^{\prime}+\sum_{i=1}^{n} y_{i}^{2}
\end{array}\right]
$$

where $\nu_{0}^{\prime}=\nu_{0}-k-1=\nu_{0}-3$. Provided that the sample variance is approximately equal to the prior variance, i.e.

$$
\sum_{i=1}^{n} x_{i}^{2} \approx n \sigma_{X}^{2}=n \quad \sum_{i=1}^{n} y_{i}^{2} \approx n \sigma_{Y}^{2}=n
$$


our expression for $\Sigma_{n}$ becomes:

$$
\left[\begin{array}{cc}
1 & \rho_{n} \\
\rho_{n} & 1
\end{array}\right]
$$

where

$$
\rho_{n}=\frac{\nu_{0}^{\prime} \rho_{0}+\sum_{i=1}^{n} x_{i} y_{i}}{\nu_{0}^{\prime}+n}
$$

Equation (12) is the covariance matrix of a bivariate normal with unit marginal variances and correlation coefficient $\rho_{n}$. For vacuous prior correlation, $\rho_{0} \in$ $[-1,1]$, we thus get the following posterior bounds on the correlation:

$$
\begin{gathered}
\underline{\rho}_{n}=\frac{-\nu_{0}^{\prime}+\sum_{i=1}^{n} x_{i} y_{i}}{\nu_{0}^{\prime}+n} \\
\bar{\rho}_{n}=\frac{\nu_{0}^{\prime}+\sum_{i=1}^{n} x_{i} y_{i}}{\nu_{0}^{\prime}+n}
\end{gathered}
$$

provided that our observations, and our prior, have unit variance and zero mean (which we can achieve by linear transformation without loss of generality).

This analysis generalises almost trivially to cases with more than two variables, $k>2$-we leave this to the reader.

\subsection{Application to body mass index example}

We now illustrate our model on the evaluation of the body mass index $R=$ $X / Y^{2}$, where $X$ is a person's weight in kilograms and $Y$ is his/her height in meters. The body mass index is commonly used to detect under- and overweight. We aim (1) to assess the dependence between $X$ and $Y$ in a particular population, and (2) to extract a robust inference about $R$ in this population.

We consider 30 paired observations of heights and weights of 11 years old girls [5, p. 75]. The weight $X$ has sample mean $\bar{x}=36.2$, sample standard deviation $s_{X}=7.7$, with no strong evidence against normality (p-value ${ }^{3} 0.017$ ). The height $Y$ has sample mean $\bar{y}=1.448$, sample standard deviation $s_{Y}=0.077$, with no evidence against normality whatsoever (p-value 0.711 ). We will assume that $X$ and $Y$ have known means, equal to $\bar{x}$ and $\bar{y}$. We also assume that, a priori, $\sigma_{X}=7.7$ and $\sigma_{Y}=0.077$ in eq. (9), but we are vacuous about the prior correlation. For reference, it may be useful to note that the sample correlation between $X$ and $Y$ is in fact 0.742 . For the sake the example, we assume that the sample is drawn from a bivariate normal distribution, although there is reasonably strong evidence against joint normality (p-value 0.00388 ).

Figure 1 shows the bounds on the correlation of the posterior covariance matrix in eq. (10) with $\nu_{0}^{\prime}=2$ and $\rho_{0} \in[-1,1]$. The two values converge steadily with a final interval $\left[\underline{\rho}_{30}, \bar{\rho}_{30}\right]=[0.630,0.759]$. The expectation of $R$ is bounded by $\underline{E}(R)=17.10$ and $\bar{E}(R)=17.16$. Similarly, we may wonder about the probability of $R$ to be in a "healthy" range, which is about $A=[14,19.5]$ for girls aged 11. We obtain bounds $\underline{P}(R \in A)=0.66$ and $\bar{P}(R \in A)=0.71$.

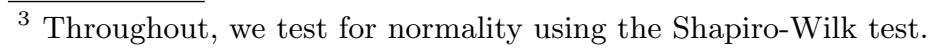




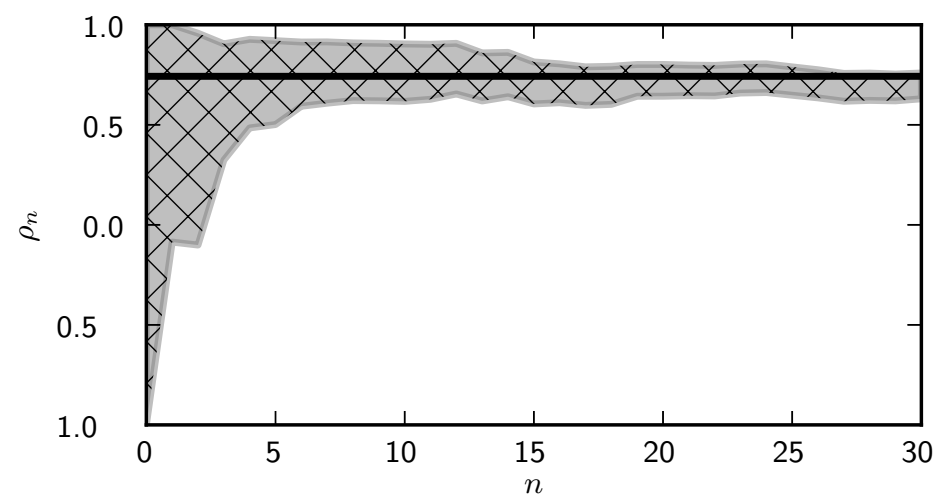

Fig. 1. Lower and upper correlation estimates $\underline{\rho}_{n}$ and $\bar{\rho}_{n}$ as a function of the sample size $n$, with $\nu_{0}^{\prime}=2$. The solid horizontal line denotes the sample correlation for $n=30$.

\section{Robust Bayesian Dependence Learning for Bivariate Categorical Data}

\subsection{The Model}

Consider a bivariate categorical random quantity $Z:=(X, Y)$ with $X$ taking values in a finite set $\mathcal{X}=\left\{1, \ldots, m_{X}\right\}$, and $Y$ taking values in a finite set $\mathcal{Y}=$ $\left\{1, \ldots, m_{Y}\right\}$. The parameters $\theta_{x}$ and $\phi_{y}$ determine the marginal distributions:

$$
p(x \mid \theta)=\theta_{x}, \quad p(y \mid \phi)=\phi_{y} .
$$

We assume that $m_{X} \geq 2, m_{Y} \geq 2, \theta_{x}>0$ and $\phi_{y}>0$.

We are interested in learning the dependence structure of $X$ and $Y$. One very general way to express the full joint distribution of $(X, Y)$ is by introducing parameters $w_{x y}$ such that

$$
p(x, y \mid \theta, \phi, w)=w_{x y} \theta_{x} \phi_{y}
$$

subject to the constraints

$$
\begin{aligned}
\sum_{x \in \mathcal{X}} \sum_{y \in \mathcal{Y}} w_{x y} \theta_{x} \phi_{y} & =1 \\
\sum_{x \in \mathcal{X}} w_{x y} \theta_{x} & =1 \\
\sum_{y \in \mathcal{Y}} w_{x y} \phi_{y} & =1
\end{aligned}
$$


Equations (19) and (20) simply follow from

$$
\begin{aligned}
& \sum_{x \in \mathcal{X}} w_{x y} \theta_{x} \phi_{y}=\sum_{x \in \mathcal{X}} p(x, y \mid \theta, \phi, w)=p(y \mid \theta, \phi, w)=\phi_{y} \\
& \sum_{y \in \mathcal{Y}} w_{x y} \theta_{x} \phi_{y}=\sum_{y \in \mathcal{Y}} p(x, y \mid \theta, \phi, w)=p(x \mid \theta, \phi, w)=\theta_{x}
\end{aligned}
$$

respectively. This model specification is over-parametrized, but it allows us to model the marginal distributions and the dependence structure separately, where the matrix $w$ plays precisely a similar role as a copula in general bivariate models for (usually) continuous random quantities. However, a key difference and major difficulty with the above model is that the constraints on $w$ depend on $\theta$ and $\phi$ : the separation is thus not as complete as with copulas, where dependence structure is parametrised independently of the marginals. For this reason, it seems most natural to consider a two-stage situation where we first learn about the marginal parameters $\theta$ and $\phi$, followed by learning about the dependence structure $w$ conditional on what we learnt about $\theta$ and $\phi$.

\subsection{Inference for Known Marginals}

For this reason, as a stepping stone towards general inference about $\theta$, $\phi$, and $w$, here we consider a scenario where the marginal distributions are already fully known, and we only aim at inference about $w$. While this may appear somewhat restrictive, and perhaps even artificial, there are practical scenarios where one has very substantial information about the probability distributions for the random quantities $X$ and $Y$ separately, but relatively little information about their joint distribution.

There are $\left(m_{X}-1\right)\left(m_{Y}-1\right)$ degrees of freedom for the components of $w$. In case data is limited, to enable sufficiently useful inference, it seems natural to assume a reduced-dimensional parametric form for $w$, which may naturally correspond to an (assumed) ordering of the categories, as we will illustrate in section 3.3. Let $n_{x y}$ denote the number of observations of $(X, Y)=(x, y)$, with total number of observations $n=\sum_{x \in \mathcal{X}} \sum_{y \in \mathcal{Y}} n_{x y}$ and row and column totals denoted by $n_{x *}=\sum_{y \in \mathcal{Y}} n_{x y}$ and $n_{* y}=\sum_{x \in \mathcal{X}} n_{x y}$, respectively. So, there are $n_{x *}$ observations of $X=x$ and $n_{* y}$ observations of $Y=y$.

Without further restrictions on $w$, it seems tempting to fit the model to match the non-parametric maximum likelihood estimate

$$
\hat{p}(x, y \mid w)=\frac{n_{x y}}{n}
$$

by setting

$$
\hat{w}_{x y}=\frac{n_{x y}}{n \theta_{x} \phi_{y}} .
$$

A problem is that this estimate will usually violate eqs. (19) and (20). For instance,

$$
\sum_{x \in \mathcal{X}} \hat{w}_{x y} \theta_{x}=\sum_{x \in \mathcal{X}} \frac{n_{x y}}{n \theta_{x} \phi_{y}} \theta_{x}=\frac{n_{* y}}{n \phi_{y}} \neq 1
$$


as soon as $\frac{n_{* y}}{n} \neq \phi_{y}$. A proper maximum likelihood estimate would maximize the likelihood subject to all constraints embodied by eqs. (18) to (20). Solving this optimisation problem poses an interesting challenge.

Bayesian inference for $w$ will face a similar challenge: $w$ lives in a convex subspace of $\mathbb{R}^{m_{X} \times m_{Y}}$ determined by eqs. (18) to (20). Application of Bayes's theorem requires integrating (or simulating) over this space. Nevertheless, the basic principles behind Bayesian inference for $w$ are simple, and sensitivity analysis is similar to the imprecise Dirichlet model [14]. Specific dimension-reduced models, where we have a much better handle on the parameter space, will be illustrated in more detail in section 3.3.

The likelihood is given by

$$
\prod_{x \in \mathcal{X}} \prod_{y \in \mathcal{Y}}\left(w_{x y} \theta_{x} \phi_{y}\right)^{n_{x y}}
$$

so as conjugate prior we can choose

$$
f\left(w \mid \alpha_{0}\right) \propto g(w) \prod_{x \in \mathcal{X}} \prod_{y \in \mathcal{Y}}\left(w_{x y} \theta_{x} \phi_{y}\right)^{\alpha_{0 x y}}
$$

where $\alpha_{0 x y}>0$ and $g$ is some arbitrary non-negative function (as long as the right hand side integrates to a finite value). With $\nu_{0}:=\sum_{x y} \alpha_{0 x y}$, this prior distribution can be interpreted as reflecting prior information equivalent to $\nu_{0}$ observations of which $\alpha_{0 x y}$ were $(X, Y)=(x, y)$. The corresponding posterior distribution is clearly $f\left(w \mid \alpha_{n}\right)$ with $\alpha_{n x y}=\alpha_{0 x y}+n_{x y}$.

Sensitivity analysis on this model could then follow an approach similar to Walley's imprecise Dirichlet model [14], by taking the set of all prior distributions for a fixed value of $\nu_{0}$. In case of an informative set of prior distributions, one may also allow the value of $\nu_{0}$ to vary within a set to allow prior-data conflict modelling [15].

As already mentioned, the remaining key difficulty is to integrate the conjugate density over the parameter space. For this reason, in the next section, we consider a reduced model.

\subsection{Reduced Model}

As a first and basic example of a reduced parametric form, consider the case $\mathcal{X}=\mathcal{Y}=\{1,2,3\}$ with known $\theta_{x}=\phi_{y}=1 / 3$ for all $x$ and $y \in\{1,2,3\}$. If the categories are ordered in some natural manner, then it might be quite reasonable to specify

$$
w=\left[\begin{array}{ccc}
1+2 \alpha & 1-\alpha & 1-\alpha \\
1-\alpha & 1+2 \alpha & 1-\alpha \\
1-\alpha & 1-\alpha & 1+2 \alpha
\end{array}\right]
$$

with $\alpha \in[0,1]$. It is easily verified that this model satisfies eqs. (18) to (20): the full matrix sums to 9 , and each of the rows and colums sum to 3 .

Note that there is no logical requirement to avoid $\alpha \in[-1 / 2,0)$, we just use this small example as an illustration. In this model, $\alpha=0$ corresponds 
to full independence between $X$ and $Y$, whereas $\alpha=1$ corresponds to perfect correlation $X=Y$. Therefore, this corresponds to a scenario where we may suspect positive correlation between $X$ and $Y$, but we are unsure about the strength of correlation. Note that the actual model reduction is achieved by additionally assuming that $X=Y=x$ has the same probability for all $x \in$ $\{1,2,3\}$, and similar for $X=x \cap Y=y$ for all $x \neq y$.

With these assumptions, statistical inference is concerned with learning about the parameter $\alpha \in[0,1]$. The likelihood function is

$$
(1+2 \alpha)^{t}(1-\alpha)^{n-t}
$$

with $t=n_{11}+n_{22}+n_{33}$ and $n=\sum_{x y} n_{x y}$ as before. The maximum likelihood estimate is

$$
\hat{\alpha}= \begin{cases}\frac{3 t-n}{2 n} & \text { if } 3 t \geq n, \\ 0 & \text { otherwise. }\end{cases}
$$

For a Bayesian approach to inference for this model, we can define a conjugate prior

$$
f\left(\alpha \mid \nu_{0}, \tau_{0}\right) \propto(1+2 \alpha)^{\tau_{0}}(1-\alpha)^{\nu_{0}-\tau_{0}}
$$

with $\tau_{0} \in\left[0, \nu_{0}\right]$, with the possible interpretation as reflecting prior information which is equivalent to $\nu_{0}$ observations of which $\tau_{0}$ have $X=Y$.

The posterior distribution is simply $f\left(\alpha \mid \nu_{0}+n, \tau_{0}+t\right)$. Sensitivity analysis is again straightforward by taking the set of prior distributions consisting of all with for example $\tau_{0} \in\left[0, \nu_{0}\right]$ for a fixed $\nu_{0}$. For instance, we would get the following robust estimate for the posterior mode of $\alpha$ :

$$
\hat{\alpha}_{n}=\left[\frac{3 t-\nu_{0}-n}{2\left(\nu_{0}+n\right)}, \frac{3 t+2 \nu_{0}-n}{2\left(\nu_{0}+n\right)}\right]
$$

when $3 t \geq \nu_{0}+n$, with similar formulas when $3 t<\nu_{0}+n$ (truncating negative values to zero).

\subsection{Application to body mass index example}

To apply the categorical model to our data, we must first discretize them, with the ordering of the categories following the ordering of natural numbers. To obtain three categories with uniform marginals, we simply discretized the $99 \%$ prediction intervals of each Gaussian marginals of section 2.2, obtaining $\mathcal{X}=$ $\{[17,32],[32,39],[39,56]\}$ and $\mathcal{Y}=\{[1.24,1.41],[1.41,1.47],[1.47,1.64]\}$.

Figure 2 shows the bounds on the posterior mode $\hat{\alpha}_{n}$ in eq. (32), with $\nu_{0}=2$. The results are similar to those obtained in section 2.2 , showing that even this very simple discretized model can capture the correlation between $X$ and $Y$, with the bounds on $\hat{\alpha}_{30}$ being $[0.56,0.66]$. From these values and the bounds of the categories, we can easily obtain bounds on the expectation of $R: \underline{E}(R)=12.9$ and $\bar{E}(R)=22.4$, which gives a much wider interval than in section 2.2. This is due to the very rough discretization: a finer discretization would likely provide a narrower interval. The lower and upper probabilities of $A=[14,19.5]$ are this time $\underline{P}(R \in A)=0$ and $\bar{P}(R \in A)=0.96$, which are almost vacuous and again show that to have meaningful inferences, a finer discretization is needed. 


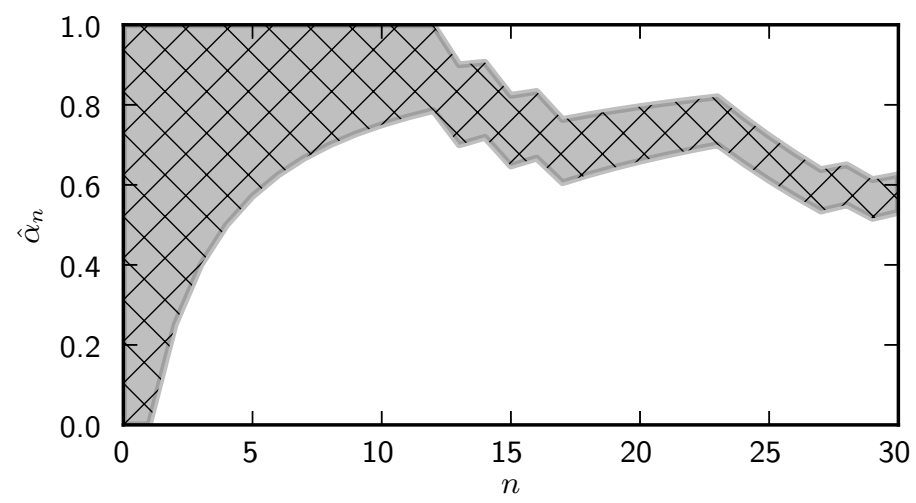

Fig. 2. Bounds on $\hat{\alpha}_{n}$ as a function of the sample size $n$, with $\nu_{0}=2$.

\section{Conclusion}

In this paper, we have introduced two preliminary models - a continuous one and a discrete one - to model dependence when joint data is limited, but assuming that the marginals are precisely known. The continuous model focused on the very special multivariate normal case. However, already in our simple example, we have seen that joint normality is rarely satisfied in practice. A major challenge is to provide methods for dependence modelling, that are both flexible and computationally tractable, whilst still producing useful inferences.

Even though the models and example studied are very preliminary, we feel that extensions of the discrete model could provide more flexibility, whilst still being easy to learn and to compute with. We see it as a promising path to learn dependency structures with imprecise probabilistic models. In particular, it can be seen as a way to approximate a continuous model, as we did in the example. In the future we plan to work on such extensions and on the identification of parametric matrices of weights more flexible than the reduced one presented here.

Finally, an obvious extension to the present work would be to relax the assumption that marginals are precisely identified, and to work with sets of marginals instead. However, this raises challenging theoretical issues, as defining a well-founded extension or equivalent formulation of Sklar's theorem for imprecise models is far from trivial.

\section{References}

1. Thomas Augustin, Frank P. A. Coolen, Gert de Cooman, and Matthias C. M. Troffaes. Introduction to imprecise probabilities. Edited book, submitted to publisher.

2. Jose M. Bernado and Adrian F. M. Smith. Bayesian Theory. John Wiley and Sons, 1994. 
3. George Boole. An investigation of the laws of thought on which are founded the mathematical theories of logic and probabilities. Walton and Maberly, London, 1854.

4. Scott Ferson, Vladik Kreinovich, Lev Ginzburg, Davis S. Myers, and Kari Sentz. Constructing probability boxes and Dempster-Shafer structures. Technical Report SAND2002-4015, Sandia National Laboratories, January 2003.

5. David J Hand, Fergus Daly, K McConway, D Lunn, and E Ostrowski. A handbook of small data sets. CRC Press, 1993.

6. Robert E Kass and Larry Wasserman. The selection of prior distributions by formal rules. Journal of the American Statistical Association, 91(435):1343-1370, 1996.

7. Roger B Nelsen. An introduction to copulas. Springer, 1999.

8. R. Pelessoni, P. Vicig, I. Montes, and E. Miranda. Imprecise copulas and bivariate stochastic orders. In Eurofuse 2013 Proceedings.

9. Erik Quaeghebeur and Gert de Cooman. Imprecise probability models for inference in exponential families. In Fabio G. Cozman, Robert Nau, and Teddy Seidenfeld, editors, ISIPTA'05: Proceedings of the Fourth International Symposium on Imprecise Probabilities and Their Applications, pages 287-296, Pittsburgh, USA, July 2005.

10. A. Sklar. Fonctions de répartition à $\mathrm{n}$ dimensions et leurs marges. Publ. Inst. Statist. Univ. Paris, 8:229-231, 1959.

11. Matthias C. M. Troffaes and Simon Blake. A robust data driven approach to quantifying common-cause failure in power networks. In F. Cozman, T. Denœux, S. Destercke, and T. Seidenfeld, editors, ISIPTA'13: Proceedings of the Eighth International Symposium on Imprecise Probability: Theories and Applications, pages 311-317, Compiègne, France, July 2013. SIPTA.

12. Matthias C. M. Troffaes and Sébastien Destercke. Probability boxes on totally preordered spaces for multivariate modelling. International Journal of Approximate Reasoning, 52(6):767-791, September 2011.

13. Peter Walley. Statistical Reasoning with Imprecise Probabilities. Chapman and Hall, London, 1991.

14. Peter Walley. Inferences from multinomial data: Learning about a bag of marbles. Journal of the Royal Statistical Society, Series B, 58(1):3-34, 1996.

15. Gero Walter and Thomas Augustin. Imprecision and prior-data conflict in generalized Bayesian inference. Journal of Statistical Theory and Practice, 3:255-271, 2009.

16. R. C. Williamson and T. Downs. Probabilistic arithmetic I: Numerical methods for calculating convolutions and dependency bounds. International Journal of Approximate Reasoning, 4:89-158, 1990. 